\title{
Changes in dietary preferences reported in pregnancy: associations with later pregnancy complications in a sample of 55,087 women
}

\section{Abstract}

Introduction

Dietary changes in pregnancy may be a marker for later pregnancy complications. This study aims to investigate if there is a difference in the proportion of women who experience specific pregnancy related complications vs those who do not, in relation to specific dietary changes made during pregnancy.

\section{Materials and methods}

Within the Danish National Birth Cohort, established during 1996 and 2003, dietary changes from 70,053 pregnancies were assessed in gestational week (GW) 25 using two open-ended questions (q1 and q2) on aversions or preferences appended to a 350-item food frequency questionnaire; "did you stop (q1) or start(q2) consuming a specific food during pregnancy?" After restricting to the firstborn child enrolled, born at term (GW 37+1-42+0) with a birth weight between 1,0 and 6,0 kilograms, excluding multiple pregnancies and abortions, dietary change answers from 55,087 women were coded into 65 food groups. Using chi- $X^{2}$ test, we compared proportions of women with and without three pregnancy complications: preeclampsia (obtained via linkage to the Danish National Patient Registry), children born small-for-gestational-age $\left(\mathrm{SGA},<10^{\text {th }}\right.$ percentile) or children born large-for-gestational-age $\left(\mathrm{LGA},>90^{\text {th }}\right.$ percentile) with respect to their answers about aversions/preferences.

\section{Results}

Among the 55,087 women, 49\% and 31\% reported any aversions or preferences, respectively. Most frequent were women reporting aversion of alcohol $(22.7 \%)$ or coffee $(15.4 \%)$, and preference of milk products $(7.9 \%)$ or fruits and berries $(6.9 \%)$, which were selected a priori for analyses. $8.3 \%$ of women experienced preeclampsia, and $9.7 \%$ and $9.3 \%$ of children were characterizes as being born SGA or LGA, respectively. Compared with no dietary change: avoiding alcohol or coffee was associated with smaller proportion of SGA $(10 \%$ vs $8 \%, p=0.01$ and $10 \%$ vs $7 \%, p<0.0001)$ and avoiding coffee was also associated with greater proportion of LGA $(9 \%$ vs $11 \%$, $p=0.01)$. Increasing milk products or fruit/berries was associated with smaller proportions of SGA (14\% vs $10 \%$ in both conditions, $p$ $<0.009)$. No significant association in reported aversions/preferences was detected for preeclampsia.

\section{Discussion}

The results lead to additional questions, such as "Why do women who increase consumption of milk products or fruits/berries tend to deliver SGA infants and why do women who stop drinking coffee tend to deliver LGA infants?" The presented results and further analyses are important to public health initiatives for optimizing maternal nutrition with long-term influence to offspring Health.

\section{Conflict of Interest}

There is no conflict of interest 